\title{
Tyrosine Photophysics During the Early Stages of Beta-Amyloid Aggregation Leading to Alzheimer's.
}

Olaf J. Rolinski ${ }^{* 1}$, Thorben Wellbrock ${ }^{1}$, David J.S. Birch ${ }^{1}$, and Vladislav Vyshemirsky ${ }^{2}$

${ }^{1}$ Photophysics Group, Centre for Molecular Nanometrology, Department of Physics, Scottish

Universities Physics Alliance, University of Strathclyde, 107 Rottenrow, Glasgow G4 0NG, UK.

${ }^{2}$ School of Mathematics and Statistics, University of Glasgow, Glasgow G12 8QQ, UK.

*Corresponding author. E-mail: o.j.rolinski@strath.ac.uk 
ABSTRACT: We have monitored the formation of toxic beta-amyloid oligomers leading to Alzheimer's disease by detecting changes in the fluorescence decay of intrinsic tyrosine. A new approach based on the non-Debye model of fluorescence kinetics resolves the complexity of the underlying photophysics. The gradual disappearance of non-monotonic fluorescence decay rates, at the early stages of aggregation as larger, tighter-packed oligomers are formed, is interpreted in terms of tyrosine-peptide dielectric relaxation influencing the decay. The results demonstrate the potential for a new type of fluorescence lifetime sensing based on dual excited-state/dielectric relaxation, with application across a broad range of biological molecules.The results also reconcile previously conflicting models of protein intrinsic fluorescence decay based on rotamers or dielectric relaxation by illustrating conditions under which both are manifest.

\section{TOC GRAPHICS}

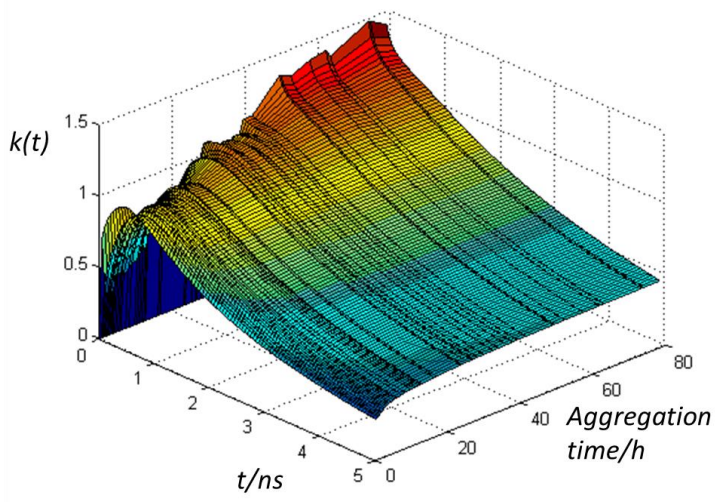

KEYWORDS. Beta-amyloid aggregation, protein fluorescence, tyrosine, non-Debye kinetics, Alzheimer's disease. 
The aggregation of misfolded protein is associated with certain diseases such as Alzheimer's and Parkinson's, where plaques of fibrils are found in the brain tissues of sufferers, although it is believed that it is the smaller precursor oligomers which damage cell walls and lead to cell death. Therefore, understanding how protein aggregation begins and how local environments affect the aggregation pathways can have significant implications in both the prevention of the disease and the development of therapeutic strategies.

One of the experimental methods helping to understand the onset of protein aggregation is timeresolved fluorescence spectroscopy of intrinsic fluorophores, which is unique in offering sub-nm spatial and sub-ns time resolution, allowing determination of the slightest changes in the fluorophore surroundings without perturbing the native structure by using extrinsic probes. The three fluorescent aminoacids tryptophan (Trp), tyrosine (Tyr) and phenylalanine, which frequently play a crucial role in protein activity, offer natural fluorescence sensors, and a noninvasive approach to protein research.

Recently, we have successfully applied this strategy to detect the early stages of Alzheimer's beta-amyloid peptide aggregation ${ }^{1-4}$. The lack of $\operatorname{Trp}$ in $A \beta_{1-40}$ conveniently eliminates the common complication of Tyr-Trp energy transfer, such that the fluorescence decay of $\operatorname{Tyr}_{10}$ in $\mathrm{A} \beta_{1-40}$ responds only to environmental change during the critical early stages of peptide oligomerisation when excited with a pulsed source at $279 \mathrm{~nm}^{5}$.

Protein fluorescence decay is usually complex and traditionally fitted to multi-exponential functions, which are explained by means of two contradictary views of the excited-state processes: one based on amodel of dielectric relaxation and the other on a model based on rotamers. Our fluorescence ${ }^{1-4}$ and molecular dynamics-based ${ }^{3}$ beta-amyloid studies to date have 
demonstrated, that a 4-rotamer model of Tyr kinetics is sufficient to explain both the Tyr fluorescence decay and the preferential orientations of Tyr in respect to the peptide backbone. However, satisfying a goodness-of-fit criterion (e.g. $\chi^{2}$ ) by increasing the number of exponentials, may lead to a false conclusion on the nature of the decay, as the multi-exponential function can be fitted to any experimental decay curve, providing the number of components is high enough.

Therefore, in order to reveal more detailed information on the fluorescence decay of Tyr in $\mathrm{A} \beta_{1 \text { - }}$ 40, we have searched for a more specific model of the decay that is parametrized by less variables than exponential treatments, thus reflecting the uniqueness of the Tyr environment and its evolution during aggregation.

Recently we have demonstrated for Trp in human serum albumin ${ }^{6}$, that the fluorescence decay of the intrinsic fluorophore in a protein can be described by the decay function

$$
I_{\alpha, \kappa}(t)=\exp \left[-\frac{1}{\kappa} \int_{0}^{\kappa\left(t / \tau_{0}\right)^{\alpha}}(1-\exp [-1 / x]) d x\right]
$$

where $\tau_{0}$ is the fluorescence lifetime of $\operatorname{Trp}$ and the pair $\alpha, \kappa$ characterises the distribution of fluorescence transition rates. This model is an adaptation of the theory describing dipole kinetics in solid materials ${ }^{7}$ and provides a more general description of fluorescence decay than has hitherto been reported and is particularly useful in helping to understand heterogeneous environments. Indeed, the exponential $(\alpha=1, \kappa=0)$ and non-exponential decay models like stretched-exponentials $^{8} \sim \exp \left[-(\mathrm{t} / \tau)^{\alpha}\right](\alpha<1, \kappa=0)$ and Becquerel functions ${ }^{9}(1+\kappa(\mathrm{t} / \tau))^{-1 / \kappa}$ $(\alpha=1, \kappa>0)$ that are in widespread use are but special cases of eq (1). 
In this letterr we report the study of $A \beta_{1-40}$ fluorescence kinetics by means of a range of more specific decay models which consider effects relevant to heterogeneous samples including the increased presence of scattered light in the experimental decay as aggregation proceeds, more general i.e. non-Debye fluorescence relaxation kinetics, and analyse the potential contribution of solute-solvent dielectric relaxation.

The commercial DAS6 reconvolution software (Horiba IBH Jobin Yvon Ltd) uses the traditional $\operatorname{approach}^{10,11}$, which does not directly include the presence of scattered light in the fluorescence signal. Using this software we have shown, that a 4-exponential model decay function is needed to describe the experimental decay (Fig.1.a and b). The values of $\chi^{2}$ (Fig.1.c) are usually in the range of 1.0-1.1, except for the decays taken between the $12^{\text {th }}$ and the $30^{\text {th }}$ hour of aggregation. Three recovered decay components $\sim 150 \mathrm{ps}, \sim 1 \mathrm{~ns}$ and $\sim 3.4 \mathrm{~ns}$ are constant during the whole process, while the longest lifetime gradually decreases from 18 ns to about 6 ns after 80 

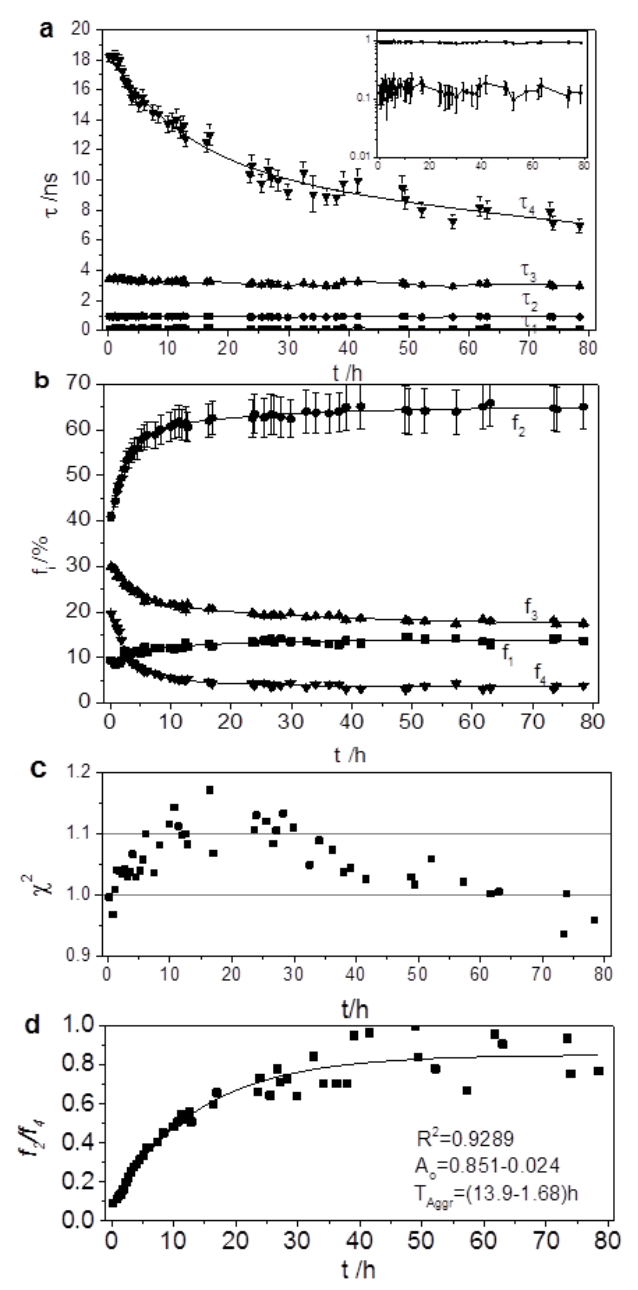

Figure.1. Parameters obtained from fitting Tyr fluorescence decay in a $50 \mu \mathrm{M} A \beta_{1-40}$ solution to a 4 exponential decay model as aggregation proceeds: (a) Tyr fluorescence decay times, (b) percentage fluorescence decay contributions, (c) the $\chi^{2}$ values, and (d) the $f_{2} / f_{4}$ ratio between fluorescence decay fractional contributions. The experimental errors shown are 3 standard deviations.

hours. This result is consistent with the existence of four Tyr rotamers, with the longest decay time associated with a rotamer being increasingly quenched as the aggregation progresses. This is in agreement with our previous report ${ }^{3}$ based on Maximum Entropy Method (MEM) analysis. 
The percentage contributions of the rotamers' fluorescence is given by eq (2) and evolve in time, indicating

$$
f_{i}=\frac{b_{i} \tau_{i}}{b_{1} \tau_{1}+b_{2} \tau_{2}+b_{3} \tau_{3}+b_{4} \tau_{4}}
$$

conformational changes of amyloids during aggregation. These changes offer an opportunity for sensing the process, for example the $f_{2} / f_{4}$ vs. time dependence (Fig $1 \mathrm{~d}$ ) provides a calibration curve, which is especially sensitive at the early stages of aggregation. This sensitivity is an important feature of our approach, as the small oligomers are currently regarded as the most toxic and monitoring the early stages of their aggregation in the presence of interfering compounds (e.g. metal ions like $\mathrm{Cu}^{2+}$ ) may help to find compounds that prevent aggregation before oligomers reach the size when they become toxic.

To investigate the potential effect of the presence of scattered excitation light in the Tyr decay, we re-analysed the raw data with a newly developed reconvolution program (see Supporting Information). Figure 2 presents the results of fitting to a 3 -exponential function. The relevant $\chi^{2}$ values (Fig.2.c.) show a similar trend to that obtained for the 4-exponential DAS6 fitting. It is worth noting, that trying the 4-exponential function with the scattered light component shows the symptom of over-parameterization, i.e. the appearance of extremely short/long decay times or pairs of decay components of the same or very similar values, thus a 3-exponential function is found to be most meaningful in this analysis. 

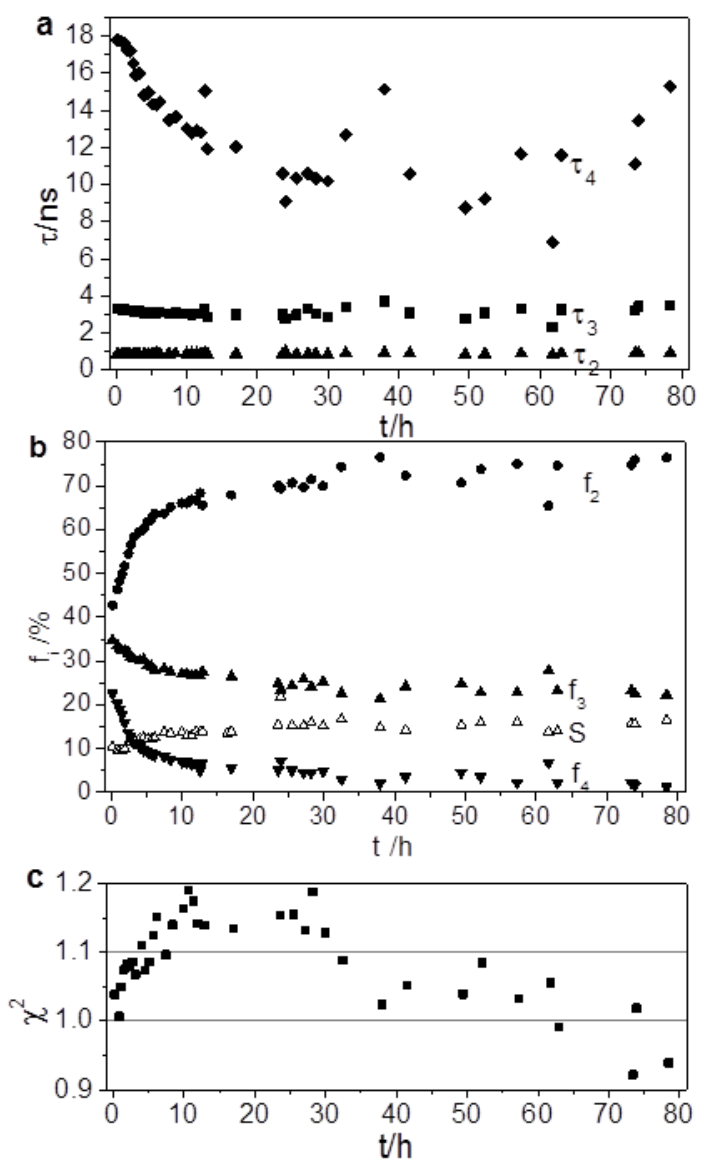

Figure.2. Parameters obtained from fitting Tyr decay in a $50 \mu \mathrm{M} A \beta_{1-40}$ sample, to a 3 exponential decay model with scattered light included as aggregation proceeds: (a) Tyr fluorescence decay times, (b) percentage contributions of the fluorescence decay components and the scattered excitation light $S$, (c) the $\chi^{2}$ values.

The first thing to note in Fig. 2 is that $\mathrm{S}$ increases with time, which is intuitively consistent with oligomer aggregation. Comparing Fig.2a and b with Fig.1a and b shows that the recovered parameters are very similar to the decay times and percentage contributions obtained for the 4exponential DAS6 fitting, except for the shortest decay component and its contribution. Moreover, the percentage contributions of the scattered light (open triangles in the Fig.2b.) 
overlap with the shortest decay contributions in Fig.1.b, indicating, that the shortest decay component identified in the DAS6 fitting can be equally well described by the addition of scattered excitation light. It is worth noting, that regardless of which option is more appropriate, a plot of $f_{2} / f_{4}$ ratio vs. aggregation time (Fig.1d) remains a good measurand for identifying the stage of $A \beta_{1-40}$ oligomerisation and by this mean the multi-exponential fitting provides useful sensing data.

Nevertheless, given the ambiguities implicit in exponential analyses an alternative approach based on the eq. (1) has been applied here in order to glean further information on the underlying photophysics. Fig.3. shows the recovered values of $\tau, \alpha$ and $\kappa$ and the relevant $\chi^{2}$ values (full circles in Fig.3c, which are compared with the $\chi^{2}$ for 4-exp DAS6 analysis (open triangles) and 3-exp approach including scattered light (open circles)). Although the $\chi^{2}$ values obtained for non-Debye model are usually slightly higher than those for multi-exponential functions, the nonDebye approach might be still regarded as the most adequate for the measured decay. This is because the appropriateness of the model decay has to be assessed on the basis of the $\chi^{2}$ value and the number of parameters $k$ of the model function. This can be assessed in terms of the Akaike Information Criterion $(A I C),{ }^{12} A I C=\chi^{2}+2 k, \mathrm{t}$ The smaller value of AIC indicating the model which most adequately describes the data. 

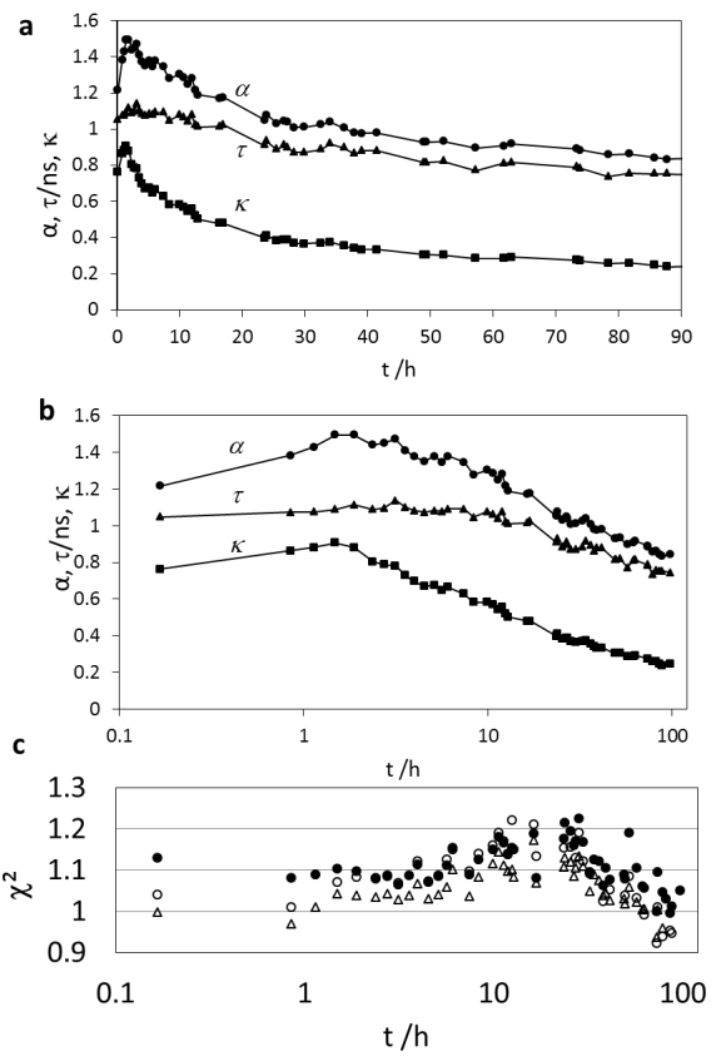

Figure.3. Parameters obtained from fitting Tyr decay in a $50 \mu \mathrm{M} A \beta_{1-40}$ sample, to a nonDebye decay model as aggregation proceeds: (a) $\alpha, \tau$ and $\kappa$ parameters, (b) same as (a) but in the logarithmic time scale, (c) the $\chi^{2}$ values for the non-Debye model (full circles), compared with the $\chi^{2}$ values obtained for the 4-exponential (triangles) and 3-exponential (open circles) models.

Consequently, a substantial improvement in the $\chi^{2}$ value is needed to justify using a model of higher complexity and number of parameters. In our case, the number of physical parameters in a non-Debye model is $k=3(A I C \approx 7)$, while for the 3 - and 4-exponential models we have $k=5$ $(A I C \approx 11)$ and $k=7(A I C \approx 15)$, respectively.

Fig. $3 b$, which plots the results in a logarithmic time scale for better clarity, shows that $\alpha$ and $\kappa$ parameters initially increase for the first 3 hours of the aggregation and then they start to drop, 
while $\tau$ remains constant for about 12 hours, and then starts to decrease. These results divide the aggregation process into three stages: 0-3 hours ( $\alpha$ and $\kappa$ increase, $\tau$ constant), 3-12 hours ( $\alpha$ and $\kappa$ decrease, $\tau$ constant) and 12-90 hours ( $\alpha, \kappa$ and $\tau$ decrease).

The fitted values of the parameter $\alpha$ are fundamental to a proper understanding of the measured decay. The original general model of excited state relaxation resulting in eq. (1) for the fluorescence decay assumes ${ }^{6,7}$ that the probability that fluorophore remains in the excited state at a time $t$ after excitation is $\exp [-k t]$, when the rate $k$ of the decay is a subject of the $\alpha$-stable distribution $f_{\alpha}(k)$, where $0<\alpha \leq 1$ is the so called index of stability. The special case $\alpha=1$ indicates an exponential decay and, in any case, the index of stability cannot be higher than 1 . The experimentally obtained $\alpha>1$, observed for at least first 40 hours of amyloid aggregation, implies that the detected photophysics is more complex than that proposed by the model decay function given by eq. (1) and some additional effects are involved. Nevertheless, the good $\chi^{2}$ values obtained for eq. (1) characterized by only 3 parameters encouraged us to investigate the potential causes for $\alpha>1$. For this reason, we have considered the time dependence of the rate of decay $k(t)$ for the fluorescence decay described by eq.1. The relationship between any fluorescence decay $I(t)$ and the relevant decay rate $k(t)$ is

$$
\frac{d I(t)}{d t}=-k(t) I(t)
$$

thus for eq. (1)

$$
k(t)=-\frac{d \ln I(t)}{d t}=-\frac{\alpha}{\tau_{0}}\left(\frac{t}{\tau_{0}}\right)^{\alpha-1}\left(1-\exp \left[-\frac{1}{\kappa}\left(\frac{t}{\tau_{0}}\right)^{-\alpha}\right]\right)
$$


The parameters presented in Fig. 3 were used to plot $k(t)$ for the decays measured at different times of aggregation (Fig.4.).

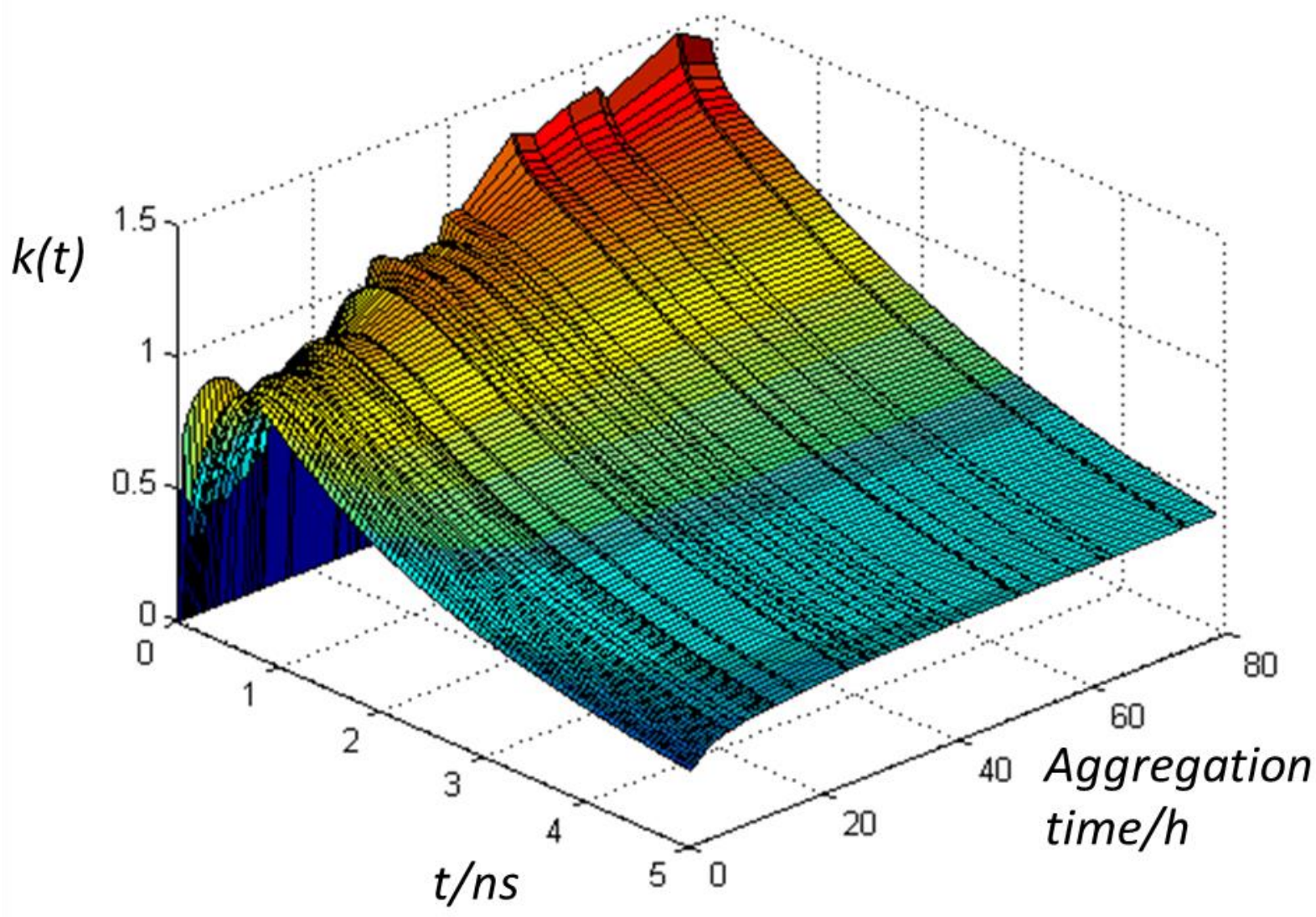

Figure.4. The time-dependent rates of the decay $k(t)$ of the Tyr at different stages of $\mathrm{A} \beta_{1-40}$ aggregation calculated according to the eq. (4).

As can be seen, the cases of $\alpha>1$ correspond to the $k(t)$ functions that show initial increase and then gradual decrease. The growing parts become shorter at later stages of aggregation and finally disappear after about 40 hours, which is the point when $\alpha$ becomes less than 1 . In the 
decay measured at later times $\alpha$ decreases further, indicating that the kinetics becomes more consistent with the model of fluorescence relaxation as per eq.(1).

The above observations allow us to hypothesise that the reason for the $\alpha>1$ (and consequently the increases of the $k(t)$ observed in the initial hours of aggregation) is a dielectric relaxation of tyrosine within the oligomers and its local environment, causing a time-dependent Stokes shifts of the emission spectra.

To investigate this hypothesis, we have simulated the decays according to the Toptygin-type approach $^{13,14}$, and then fitted eq. (1) to these decays. The parameters used for this simulation are not related to the Tyr fluorescence features. It has been assumed, that the wavenumber- and timedependent fluorescence intensity $I(v, t)$ is ${ }^{13,14}$

$$
I(v, t)=a v^{3} S\left(v-v_{c}(t)\right) I(t)
$$

where $S(v)$ is the vibration envelope representing the fluorescence transitions, $I(t)$ is a single exponential fluorescence decay with the lifetime $\tau_{F}=1 \mathrm{~ns}$ and $v_{c}(t)$ is the time variation of the spectral centre of gravity. $v_{c}(t)$ shifts in time exponentially from the initial $v_{0}$ to the final $v_{\infty}$ value with the solute-solvent relaxation time $\tau_{r}$, i.e.

$$
v_{c}(t)=v_{\infty}+\left(v_{0}-v_{\infty}\right) \exp \left[-t / \tau_{r}\right]
$$

The assumed wavenumbers were $v_{0}=35333 \mathrm{~cm}^{-1}, v_{\infty}=28303 \mathrm{~cm}^{-1}$, and $S(v)=\exp \left[-v^{2} / 2 \sigma^{2}\right]$ was a Gaussian distribution with $\sigma=3500 \mathrm{~cm}^{-1}$. The time-dependent emission spectra corresponding to these parameters are illustrated in Fig.5. 


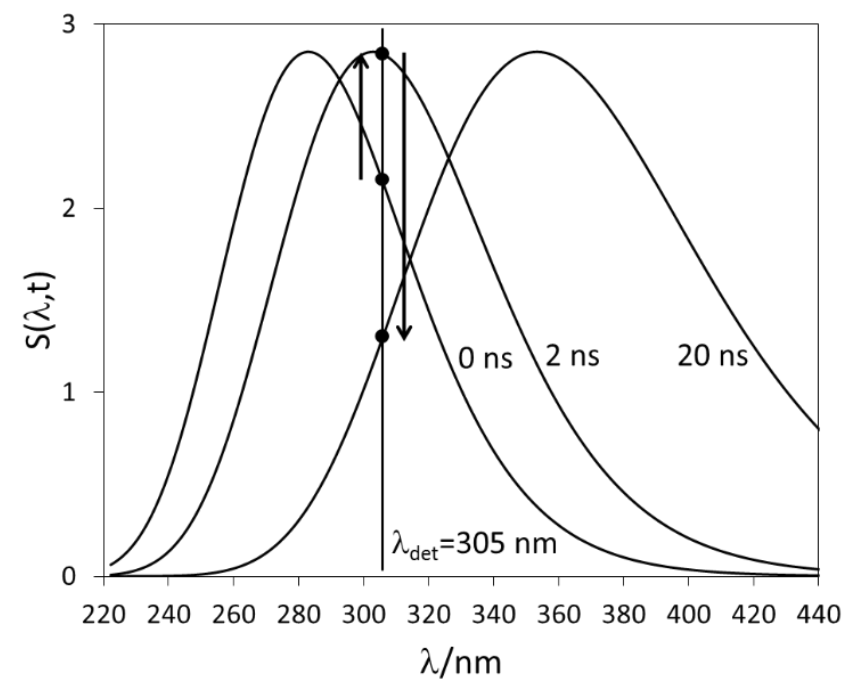

Figure.5. The time-dependent emission $\operatorname{spectra} S(\lambda, t)$ at different times after excitation. The plot explains the initial growth of the $k(t)$ as the result of dielectric relaxation with the solutesolvent relaxation time $\tau_{r}=4 \mathrm{~ns}$. The other parameters used in the simulation are discussed in the text.

The synthetic decays were simulated for $v=32788 \mathrm{~cm}^{-1}(\lambda=305 \mathrm{~nm})$ and three solute-solvent relaxation times $\tau_{r}=0.01,4$ and $12 \mathrm{~ns}$, and then fitted to the decay using eq. (1). The recovered parameters are shown in Table 1.

\begin{tabular}{|c|c|c|c|}
\cline { 2 - 4 } \multicolumn{1}{c|}{} & \multicolumn{3}{c|}{ Fitted parameters } \\
\cline { 2 - 4 } \multicolumn{1}{c|}{} & $\tau_{r}=0.01 \mathrm{~ns}$ & $\tau_{r}=2 \mathrm{~ns}$ & $\tau_{r}=20 \mathrm{~ns}$ \\
\hline$\alpha$ & 1.006 & 1.240 & 1.040 \\
\hline$\kappa$ & 0.006 & 0.1217 & 0.001 \\
\hline$\tau$ & 1.008 & 1.172 & 1.114 \\
\hline$\chi^{2}$ & 0.837 & 0.904 & 0.939 \\
\hline
\end{tabular}

Table 1. Recovered parameters of the simulated decays fitted to the eq. (1). 
The $\chi^{2}$ values less than 1 indicate overfitting, which suggests that for the mono-exponential decay of the excited-state population $I(t)$, the decays measured in the presence of dielectric relaxation seem to be simpler than given by eq. (1). More importantly, the simulations have demonstrated that the effect of the dielectric relaxation is negligible if the solute-solvent relaxation time $\tau_{r}$ is small as compared to the mean fluorescence lifetime $\tau_{F}$, ie $\tau_{r}<<\tau_{F}$, which can be explained by all fluorescence occurring from the stable solute-solvent relaxed fluorophores and there being no readily observable Stokes shift. It is also negligible, if $\tau_{r}>>\tau_{F}$, as all fluorescence occurs from the "frozen" Franck-Condon solute-solvent state. However, in the intermediate case $\tau_{r} \approx \tau_{F}$, the emission spectrum shifts at the same time as fluorescence occurs and is an analogy of fluorescence anisotropy decay . Because the fluorescence is detected at a fixed wavelength the detected fluorescence intensity decay resulting from the excited-state relaxation is modified by an initial increase and then decrease in the intensity (for the arrangements shown on Fig.5) due to the spectrum shifting towards longer wavelengths. A combination of both effects may lead to the decay being more complex than anticipated by the model described by eq.(1).

The recovered values of the parameters confirm our hypothesis that the presence of dielectric relaxation with $\tau_{r} \approx \tau_{F}$ results in $\alpha>1$, if data is fitted to eq. (1).

To conclude, in order to obtain full information on the underlying kinetics, the wavenumber- and time-dependent Tyr fluorescence intensity, i.e. the decay model given by (5), with the I(t) part represented by eq. (1), has to be applied to the decays measured at the series of detection wavelengths for each step of aggregation. Moreover, it seems likely that the ability to separate dielectric relaxation from other decay mechanisms could prove to be a powerful tool in the 
analysis of fluorescence decay, revealing hitherto intractable aspects of such as solvent accessibility and specific local interactions in biological structures.

\section{ASSOCIATED CONTENT}

Supporting Information. Experimental: sample preparation, fluorescence decay measurements, lifetime data analysis. This material is available free of charge via the Internet at http://pubs.acs.org.

\section{AUTHOR INFORMATION}

\section{Corresponding Author}

*Email: o.j.rolinski@strath.ac.uk

\section{Notes}

The authors declare no competing financial interests.

\section{ACKNOWLEDGMENT}

OJR would like to thank Prof. Karina Weron for valuable discussions on the theoretical models of relaxation.

\section{REFERENCES}

(1) Rolinski; O.J.; Amaro, M.; Birch, D.J.S.; Early Detection of Amyloid Aggregation Using Intrinsic Fluorescence, Biosens. \&Bioelectr. 2010, 25, 2249-2252. 
(2) Amaro, M.; Birch, D.J.S.; Rolinski, O.J., Beta-Amyloid Oligomerisation Monitored by Intrinsic Tyrosine Fluorescence, PCCP 2011,13, 6434-6441.

(3) Amaro, M.; Kubiak-Ossowska, K.; Birch, D.J.S.; Rolinski, O.J.; Initial Stages of BetaAmyloid $A \beta_{1-40}$ and $A \beta_{1-42}$ Oligomerization Observed Using Fluorescence Decay and Molecular Dynamics Analyses of Tyrosine, Methods Appl. Fluoresc. 2013, 1, 015006.

(4) Amaro, M.; Wellbrock, T.; Birch, D.J.S.; Rolinski, O.J.; Inhibition of Beta-Amyloid Aggregation by Fluorescent Dye Labels, Appl.Phys.Lett. 2014, 104, 063704.

(5) McGuinness, C.D.; Sagoo, K.; McLoskey, D.; and Birch, D.J.S.; A New Sub-nanosecond LED at 280 nm: Application to Protein Fluorescence, Meas.Sci.Technol., 2004, 15,11 L19-L22.

(6) Rolinski, O.J.; Vyshemirsky, V.; Fluorescence Kinetics of Tryptophan in Heterogeneous Environment, Methods Appl. Fluoresc. 2014, 2, 045002.

(7) Weron, K.; Jurlewicz, A.; 2 Forms of Self-similarity as a Fundamental Feature of the Power-law Dielectric Response J.Phys.A:Math.Gen. 1993, 26, 395-410.

(8) Berberan-Santos M. N.; Bodunov E. N.; Valeur B.; Mathematical Functions for the Analysis of Luminescence Decays with Underlying Distributions. 1. Kohlrausch Decay Function (Stretched Exponential). Chem.Phys. 2005, 315, 171-182.

(9) Berberan-Santos M. N.; Bodunov E. N.; Valeur B.; Mathematical Functions for the Analysis of Luminescence Decays with Underlying Distributions. 2.Becquerel (Compressed Hyperbola) and Related Decay Functions. Chem.Phys. 2005, 317, 57-62.

(10) Birch, D.J.S.; Imhof, R.E.; Time Domain Fluorescence Spectroscopy Using TimeCorrelated Single Photon Counting. In: Lakowicz JR (ed) Topics of Fluorescence Spectroscopy, Vol.1. pp.1-96, Springer, New York, 2002. 
(11) Birch, D.J.S.; Chen, Y.; Rolinski, O.J.; Fluorescence. Ch.1 in: Photonics: Scientific Foundations, Technology and Applications, Vol. IV, First Edition, D.L.Andrews (ed), John Wiley \&Sons, Inc., 2015.

(12) Burnham, K.P.; Anderson, D.R.; Model Selection and Multimodel Interference: A Practical Information - Theoretic Approach, 2nd edtn,New York: Springer, 2002.

(13) Toptygin, D.; Brand, L.; Spectrally- and Time-resolved Fluorescence Emission of Indole During Solvent Relaxation: a Quantitative Model, Chem.Phys.Letts. 2000, 322, 496-502. (14) Toptygin, D.; Savtchenko, R.S.; Meadow, N.D.; Brand, L.; Homogeneous Spectrally- and Time-resolved Fluorescence Emission From Single-tryptophan Mutants of IIA ${ }^{\text {Glc }}$ protein, J.Phys.Chem.B, 2001, 105, 2043-2055. 


\section{SUPPORTING INFORMATION:}

\section{Tyrosine Photophysics During the Early Stages of}

\section{Beta-Amyloid Aggregation Leading to}

\section{Alzheimer's.}

Olaf J. Rolinski ${ }^{* 1}$, Thorben Wellbrock ${ }^{1}$, David J.S. Birch ${ }^{1}$, and Vladislav Vyshemirsky ${ }^{2}$

${ }^{1}$ Photophysics Group, Centre for Molecular Nanometrology, Department of Physics, Scottish Universities Physics Alliance, University of Strathclyde, 107 Rottenrow, Glasgow G4 0NG, UK. ${ }^{2}$ School of Mathematics and Statistics, University of Glasgow, Glasgow G12 8QQ, UK.

\section{EXPERIMENTAL}

Sample preparation. $\mathrm{A} \beta_{1-40}$ peptides were purchased from Anaspec Inc., USA.

Hexafluoroisopropanol (HFIP) and N-(2-hydroxyethyl)piperazine-N'-(2-ethanesulfonic acid) buffer solution (HEPES) were purchased from Sigma-Aldrich (Poole, UK). All compounds were used without further purification. The lyophilised $\mathrm{A} \beta_{1-40}$ protein was prepared via treatment with HFIP $(0.1 \mathrm{mM})$ and sonicated for 10 minutes to remove small aggregates. The stock solution was then aliquoted into Eppendorf microcentrifuge bottles and HFIP was let to 
evaporate. The samples were stored at $-20{ }^{\circ} \mathrm{C}$. For each experiment the sample was freshly thawed and treated with HFIP $(0.1 \mathrm{mM})$ and let to evaporate. It was then suspended in Hepes buffer (100 mM; pH 7.4) to a final concentration of $50 \mu \mathrm{M}$. The experiments were performed at $37^{\circ} \mathrm{C}$ and all components and solutions were in thermal equilibrium before use.

Fluorescence decay measurements. For fluorescence decay measurements we have used the Fluorocube fluorescence lifetime system (Horiba Jobin Yvon IBH Ltd, UK), based on the technique of time-correlated single-photon counting (TCSPC). The system was equipped with Seya-Namioka monochromators for excitation and emission. The light source used for experiments was a AlGaN pulsed light emitting diode ${ }^{5}$ with a centre wavelength of $279 \mathrm{~nm}$, pulse duration of $\sim 600 \mathrm{ps}$ (FWHM) and repetition rate of $1 \mathrm{MHz}$. The channel width was 13.47 ps. The sample holder was kept at $37{ }^{\circ} \mathrm{C}$ during all experiments and controlled by Neslab TRE-11 thermostat (Thermo Scientific UK). Fluorescence decay was measured at a wavelength of $315 \mathrm{~nm}$. The bandwidths in the excitation and emission monochromators were $3 \mathrm{~nm}$.

Lifetime data analysis. The potential effect of the scattered light in the detected signal was investigated by comparing the results obtained for fitting the multi-exponential decay using two different fitting procedures, one without and the other with a component representing the scattered light. For multi-exponential analysis without the scattered light contribution, the commercial Data Analysis Software (DAS6, IBH Horiba Ltd.) has been used. DAS6 uses the shift parameter $\Delta$ to represent the time-shift between the prompt and the fitted decay profile caused by the detector colour effect and finite time channel width ${ }^{10} . \Delta$ is iterated during the fitting procedure to obtain the best fit.

We started data analysis with a multi-exponential decay 


$$
I(t)=\sum_{i=1}^{N} b_{i} \exp \left[-t / \tau_{i}\right]
$$

where $\tau_{i}$ indicates fluorescence decay time of the $i$-th rotamer.

To obtain least-square fits to the multi-exponential and non-Debye decay models, both assuming the presence of the scattered light, a minimization search has been performed for the $\chi^{2}$ goodness of fit criterion defined as

$$
\chi^{2}(\text { par. })=\frac{1}{N-\gamma} \sum_{i=1}^{N} \frac{\left(F_{i}-P_{i}(\text { par. })\right)^{2}}{F_{i}}
$$

where par. indicates the parameters of the assumed decay function, $N$ is the number of channels analysed, $\gamma$ is the number of parameters in the model decay, $F_{i}$ the experimental decay value for channel $i$, and $P_{i}$ is the model prediction for channel $i$.

$$
P_{i}(\text { par. })=a+b L(t+\Delta)+c \int_{0}^{t} L\left(t+\Delta-t^{\prime}\right) I\left(\text { par.; } t^{\prime}\right) d t^{\prime}
$$

Here $t$ is the time corresponding to the reading from channel $i, L(t)$ is the prompt excitation function, and $a, b$, and $c$ are the background level, contribution of the scattered light and the scaling parameter, respectively. $I(t)$ represents the multi-exponential (S1) or non-Debye model. 